\title{
Approximate Conditions Replacing Thin Layers
}

\author{
Clair Poignard ${ }^{1}$, Patrick Dular ${ }^{2}$, Ronan Perrussel ${ }^{3}$, Laurent Krähenbühl ${ }^{3}$, Laurent Nicolas ${ }^{3}$, and \\ Michelle Schatzman ${ }^{4}$ \\ ${ }^{1}$ Centre de Mathématiques Appliquées, UMR CNRS 7641, École Polytechnique, F-91128 Palaiseau, France \\ ${ }^{2}$ University of Liège, F.R.S.-FNRS, Department of Electrical Engineering and Computer Science, B28, B-4000 Liège, Belgium \\ ${ }^{3}$ Laboratoire Ampère, UMR CNRS 5005, Université de Lyon, École Centrale de Lyon, Écully F-69134, France \\ ${ }^{4}$ Institut Camille Jordan, UMR CNRS 5208, and Université de Lyon, Université Lyon 1, F-69622 Villeurbanne, France
}

\begin{abstract}
We provide a rigorous asymptotic method to build approximate conditions equivalent to a thin layer. With these conditions the electromagnetic field may be computed in domains such as a biological cell, without meshing the membrane. The influence of the membrane is replaced by an appropriate condition on the inner domain, while in the thin layer, the approximate field is explicitly known. We give error estimates, which validate our asymptotic method, and we present a few numerical results performed with the finite-element method.
\end{abstract}

Index Terms-Asymptotic methods, finite-element method, thin layers.

\section{INTRODUCTION}

T HE distribution of the electromagnetic field in a biological cell is important for bioelectromagnetic investigations. For instance, a sufficiently large amplitude of the transmembranar potential (TMP), which is the difference of the electric potentials between both sides of the cell membrane, leads to an increase of the permeability of this membrane [1], [2]. This phenomenon, called electropermeabilization, has already been used in oncology and holds promises in gene therapy [3], justifying precise assessments of the TMP. Since the experimental measurements of the TMP on living cells are limited - essentially due to the thinness of the cell membrane, which is a few nanometers thick-a numerical approach is often chosen [1], [4]. However, these computations are confronted with the heterogeneous parameters of the biological cells. Actually, in the Fear and Stuchly model [5], the cell is a medium of regular shape (without any corner) composed by a homogeneous conducting cytoplasm of diameter $(l)$ of a few micrometers surrounded by a very insulating membrane a few nanometers thick $(\delta l)$ (Fig. 1).

To avoid difficulties, most of the numerical computations deal with unrealistic cells (spherical and ellipsoidal cells) without details about the accuracy of the numerical method used (typically the finite-element method) [5]. However, biological cells have nontrivial shapes and Sebastián et al. have shown that the cell geometry has a significant influence on the electric field distribution. To perform computations on realistic cell shapes by considering a conduction problem, Pucihar et al. [1] propose to replace the membrane by a condition on the boundary of the cytoplasm. This condition corresponds to a contact resistance model but the details for asserting the accuracy of their method are not given.

In this paper, we present a rigorous asymptotic method to compute the electromagnetic field in smooth domains with a thin layer. Since the proofs of our results are precisely described in [6] and [7], we present the heuristics of the method and the

Digital Object Identifier 10.1109/TMAG.2007.916154

Color versions of one or more of the figures in this paper are available online at http://ieeexplore.ieee.org.

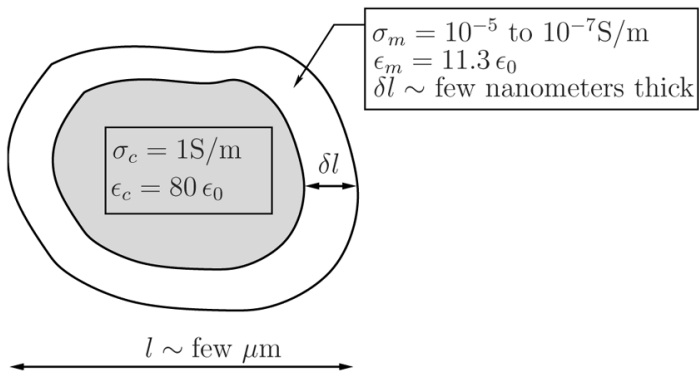

Fig. 1. Electric model of the biological cell (see Fear et al. [5])

main results, which may be useful for electromagnetic computations in biological cells and we illustrate our asymptotics with numerical simulations. For sake of simplicity, we consider a bidimensional cell; however, our method might be easily extended to three-dimensional (3-D) cells.

We deal with the quasi-static (dielectric) formulation: results for the Helmholtz equation are given in [7]. The problem is nondimensionalized: each quantity refers to a physical quantity but has no physical unit. Two cases are considered.

- An electric field (i.e., a Neumann boundary condition) is enforced directly on the exterior boundary of the membrane; we build approximate boundary conditions asymptotically equivalent to the membrane.

- The cell is embedded in a medium submitted to an electric potential; we build approximate transmission conditions.

These approximate conditions are obtained to replace the thin membrane but keeping a prescribed accuracy on the solution. To justify this accuracy, we give error estimates between the potential with the membrane and without the membrane but with the approximate conditions.

\section{APPROXIMATE BOUNDARY CONDITIONS}

\section{A. Statement of the Problem}

Denote by $\Omega$ the biological cell composed by a cytoplasm $\mathcal{O}$ surrounded by a thin membrane $\mathcal{O}_{\delta}$ with thickness $\delta$. Let $\gamma_{0}$ be the cytoplasm complex permittivity (nondimensionalized) and $\gamma_{1}$ this of the membrane. Denote by $\gamma$ the function equal to $\gamma_{0}$ in $\mathcal{O}$ and $\gamma_{1}$ in $\mathcal{O}_{\delta}$. The quasi-static formulation is

$$
\begin{cases}\nabla \cdot(\gamma \nabla V)=0, & \text { in } \Omega_{\delta} \\ \frac{\partial V}{\partial n}=\phi, & \text { on } \partial \Omega_{\delta}\end{cases}
$$


where $\phi$ is the electric field imposed on the boundary of the cell. We suppose that this Neumann boundary condition is as regular as we need and it satisfies $\int_{\partial \Omega_{\delta}} \phi(s) \mathrm{d} s=0$. So that $V$ is uniquely determined, we impose the gauge condition $\int_{\partial \mathcal{O}} V(s) \mathrm{d} s=0$.

Our method might be decomposed in three steps. The first step consists of a change of coordinates so that the thin parameter $\delta$ appears explicitly in the equations. In the second step, we choose an ansatz, supposing that the potential might be written as a formal series with respect to $\delta$. Then, by identifying the terms with the same power of $\delta$, we obtain a sequel of elementary problems. The final step is the rigorous validation of our ansatz, providing a priori error estimates of the method.

\section{B. Change of Coordinates}

Since the bidimensional medium $\mathcal{O}$ is regular, in particular the presence of corners is excluded, we may parametrize it with the curvilinear coordinate. Suppose that the length of $\partial \mathcal{O}$ equals 1. There exists a 1-periodic function $\Psi$, such that for all $x \in \partial \mathcal{O}$, there exists a unique $\theta \in[0,1]$ satisfying

$$
x=\Psi(\theta) \text {, and }\left\|\Psi^{\prime}(\theta)\right\|=1 \text {. }
$$

Denoting by $n(\theta)$ the exterior normal to $\partial \mathcal{O}$ at the point $\Psi(\theta)$, we may parametrize the thin layer $\mathcal{O}_{\delta}$ as follows:

$$
\mathcal{O}_{\delta}=\{\Phi(\eta, \theta)=\Psi(\theta)+\eta \delta n(\theta), \quad \theta \in[0,1]\}
$$

The curvature $\kappa$ of $\partial \mathcal{O}$ is then the 1-periodic function defined by

$$
\forall \theta \in[0,1], \quad \kappa(\theta)=\left\|n^{\prime}(\theta)\right\| .
$$

We denote by $\mathfrak{K}$ the curvature of $\partial \mathcal{O}$ in Euclidean coordinates:

$$
\mathfrak{K}(\mathrm{x})=\kappa \mathrm{O}^{-1}(\mathfrak{x})
$$

and we define $\varphi$ as the boundary data $\phi$ translated on $\partial \mathcal{O}$

$$
\varphi(x)=\left(\left.\phi \circ \Phi\right|_{\eta=1}\right) \circ \Psi^{-1}(x) .
$$

The main idea of our asymptotic method is to write the Laplace operator in local coordinates so that the thin parameter $\delta$ appears explicitly. Denote by $\Delta_{\eta, \theta}$ the Laplace operator written in $(\eta, \theta)$-coordinates, then using change of coordinates (2) we have

$$
\Delta_{\eta, \theta}=\frac{1}{1+\delta \eta \kappa}\left(\partial_{\eta}\left(\frac{1+\delta \eta \kappa}{\delta} \partial_{\eta}\right)+\partial_{\theta}\left(\frac{\delta}{1+\delta \eta \kappa} \partial_{\theta}\right)\right)
$$

and the operators of normal derivative $\partial_{n}$ and tangential derivative $\partial_{t}$ equal

$$
\partial_{n}=\frac{1}{\delta} \partial_{\eta}, \quad \partial_{t}=\partial_{\theta}
$$

Supposing that $V$ may be written as follows:

$$
V=V_{0}+\delta V_{1}+\cdots
$$

we consider this expression of $V$ with the Laplace operator in local coordinates (4) and we order the terms of the equations:

$$
\begin{aligned}
& \Delta\left(V_{0}+\delta V_{1}+\cdots\right)=0, \text { in } \mathcal{O} \\
& \quad \frac{1}{\delta} \partial_{\eta}^{2} V_{0}+\partial_{\eta}^{2} V_{1}+\kappa \partial_{\eta} V_{0} \\
& \quad+\delta\left(\partial_{\eta}^{2} V_{2}+\kappa \partial_{\eta} V_{1}+\partial_{\theta}^{2} V_{0}\right)+\cdots=0, \text { in } \mathcal{O}_{\delta}
\end{aligned}
$$

with transmission conditions on $\partial \mathcal{O}$ (the subscript $\partial \mathcal{O}^{-}$indicates the cytoplasm side and $\eta=0^{+}$, the membrane side)

$$
\begin{gathered}
\left.V_{0}\right|_{\partial \mathcal{O}^{-}}+\left.\delta V_{1}\right|_{\partial \mathcal{O}^{-}}+\cdots=\left.V_{0}\right|_{\eta=0^{+}}+\left.\delta V_{1}\right|_{\eta=0^{+}}+\cdots \\
\gamma_{0}\left(\left.\partial_{n} V_{0}\right|_{\partial \mathcal{O}^{-}}+\left.\delta \partial_{n} V_{1}\right|_{\partial \mathcal{O}^{-}}+\cdots\right)=\gamma_{1}\left(\left.\frac{1}{\delta} \partial_{\eta} V_{0}\right|_{\eta=0^{+}}\right. \\
\left.+\left.\partial_{\eta} V_{1}\right|_{\eta=0^{+}}+\left.\delta \partial_{\eta} V_{2}\right|_{\eta=0^{+}}+\cdots\right)
\end{gathered}
$$

and with the Neumann boundary condition on $\partial \Omega_{\delta}$

$$
\left.\frac{1}{\delta} \partial_{\eta} V_{0}\right|_{\eta=1}+\partial_{\eta} V_{1}+\cdots=\left.\phi \circ \Phi\right|_{\eta=1} \cdot
$$

\section{Elementary Problems and Error Estimates}

By identifying the terms with the same power of $\delta$ in (6), we obtain the first two terms of the asymptotic expansion of the solution $V$ of Problem (1).

- Order 0. $V_{0}$ satisfies

$$
\left\{\begin{array}{ll}
\nabla \cdot\left(\gamma_{0} \nabla V_{0}\right)=0, & \text { in } \mathcal{O}, \\
\partial_{n} V_{0}=\left(\gamma_{1} / \gamma_{0}\right) \varphi & \text { on } \partial \mathcal{O}
\end{array} .\right.
$$

- Order 1. $V_{1}$ satisfies:

$$
\left\{\begin{array}{ll}
\nabla \cdot\left(\gamma_{0} \nabla V_{1}\right)=0, & \text { in } \mathcal{O}, \\
\partial_{n} V_{1}=\left(\gamma_{1} / \gamma_{0}\right)\left(\partial_{t}^{2} V_{0}+\mathfrak{K} \varphi\right) & \text { on } \partial \mathcal{O}
\end{array} .\right.
$$

Denote by $V_{\text {app }}=V_{0}+\delta V_{1}$, we have the estimate

$$
\left\|V-V_{\mathrm{app}}\right\|_{H^{1}(\mathcal{O})} \leq C\left|\frac{\gamma_{1}}{\gamma_{0}}\right| \delta^{2}\|\varphi\| .
$$

Therefore, to approximate the potential in the cytoplasm with an accuracy of order $\delta^{2}$, we have to compute problems (7) and (8) with appropriate boundary conditions for the cytoplasm.

It may be interesting to solve only one equivalent problem with a nonclassical boundary condition. Observe that $V_{\text {app }}$ satisfies

$$
\left\{\begin{array}{l}
\nabla \cdot\left(\gamma_{0} \nabla \mathrm{V}_{\mathrm{app}}\right)=0, \\
\partial_{n} \mathrm{~V}_{\mathrm{app}}=\left(\gamma_{1} / \gamma_{0}\right)\left((1+\delta \mathfrak{K}) \varphi+\left(\delta \partial_{\mathrm{t}}^{2} \mathrm{~V}_{\mathrm{app}}-\delta^{2} \partial_{\mathrm{t}}^{2} \mathrm{~V}_{1}\right)\right)
\end{array}\right.
$$

Therefore, we denote by $\tilde{V}_{\text {app }}$ the solution to

$$
\left\{\begin{array}{ll}
\nabla \cdot\left(\gamma_{0} \nabla \tilde{V}_{\text {app }}\right)=0, & \text { in } \mathcal{O}, \\
\partial_{n} \tilde{V}_{\text {app }}-\left(\gamma_{1} / \gamma_{0}\right) \delta \partial_{t}^{2} \tilde{V}_{\text {app }}=\left(\gamma_{1} / \gamma_{0}\right)(1+\delta k) \varphi & \text { on } \partial \mathcal{O}
\end{array} .\right.
$$


Using (9), we have

$$
\left\|V-\tilde{V}_{\mathrm{app}}\right\|_{H^{1}(\mathcal{O})} \leq C\left|\frac{\gamma_{1}}{\gamma_{0}}\right| \delta^{2}\|\varphi\| .
$$

In the thin layer ${ }^{1} \mathcal{O}_{\delta}$, we have the explicit formulas of $V_{\text {app }}$ written in local coordinates:

$$
\begin{aligned}
\forall x \in \mathcal{O}_{\delta}, \quad V_{\text {app }}(x)= & V_{0}\left(\left.x\right|_{\partial \mathcal{O}}\right)+\delta V_{1}\left(\left.x\right|_{\partial \mathcal{O}}\right) \\
& +\left(\left(x-\left.x\right|_{\partial \mathcal{O}}\right) \cdot n\left(\left.x\right|_{\partial \mathcal{O}}\right)\right) \varphi\left(\left.x\right|_{\partial \mathcal{O}}\right)
\end{aligned}
$$

and we have the following estimate:

$$
\left\|V-V_{\text {app }}\right\|_{H^{1}\left(\mathcal{O}_{\delta}\right)} \leq C \delta^{3 / 2}\|\varphi\|
$$

The boundary condition satisfied by $\tilde{V}_{\text {app }}$ is called approximate boundary condition. Observe that if $\mathfrak{K}$ equals 1 , i.e., if $\mathcal{O}$ is a disk, approximate boundary condition (11) is exactly the same as boundary condition (10) of Krähenbühl and Muller [8]. Here, we validate the result by an error estimate. We may perform asymptotic expansion at any order $n \geq 0$. We emphasize that according to estimate (12), our approximate boundary condition is valid for a bounded ratio $\left|\gamma_{1} / \gamma_{0}\right|$; therefore, it may be used to compute the electric field in a biological cell. As soon as $\left|\gamma_{1} / \gamma_{0}\right|$ is of order $1 / \delta$ (or bigger), which means that the thin layer is very conducting, approximate boundary condition (11) is no more valid. We refer to [6] for a precise description of this case.

\section{Numerical Simulations}

A geometry, where no analytic solution is known, has been studied. In order to show the error due to the asymptotic expansion, we are led to compute the solution of the problem with and without the membrane; these computations are performed using the finite-element method.

A numerical validation for estimation (13) can be difficult to obtain because because it necessitates the error due to the asymptotic expansion to be greater than the discretization error. If the membrane is very thin, we have to deal with a huge number of degrees of freedom, which can prevent the use of a too thin membrane for the numerical validation. This remark fully justifies the use of the approximate conditions: by considering a thin membrane but keeping a reasonable number of degrees of freedom for the discretization, it is not possible to distinguish the solutions with and without the membrane in the inner domain.

We perform computations with GetDP [9] for an elongated cell $\Omega$. The width of the interior region $\mathcal{O}$ equals 1 . In Fig. 2 , we present the steady state potentials when the thin layer is insulating. Asymptotics (7) and (8) give the approximate potential in $\mathcal{O}$ without meshing the membrane.

In Fig. 3, we draw the error made by our asymptotics with respect to the thickness $\delta$. Observe for instance that if $\delta=10^{-2}$, the error made by our method is around $10 \%$ at the order 0 and less than $1 \%$ at the order 1 .

\footnotetext{
${ }^{1}$ Observe that $x \in \mathcal{O}_{\delta}$ equals $x=\left.x\right|_{\partial \mathcal{O}}+\left(x-\left.x\right|_{\partial \mathcal{O}}\right) \cdot n\left(\left.x\right|_{\partial \mathcal{O}}\right)$, with $\left.x\right|_{\partial \mathcal{O}}$ the orthogonal projection of $x$ on $\partial \mathcal{O}$.
}

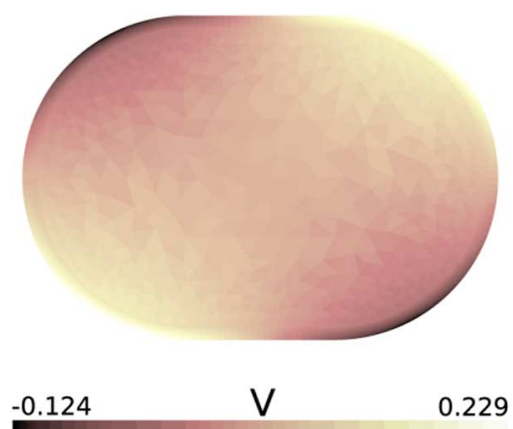

Fig. 2. Steady-state potentials in an elongated cell with an insulating membrane.

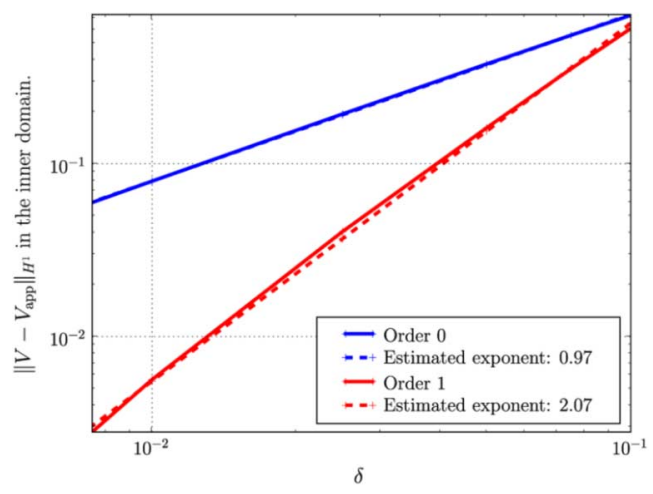

Fig. 3. Error versus the membrane thickness. $\gamma_{0}=5, \gamma_{1}=0.5$. The continuous lines are the results of the numerical computations and the dotted lines used an exponent estimated for (13) using the numerical results.

\section{APPROXIMATE TRANSMISSION CONDITIONS}

\section{A. Elementary Problems and Error Estimates}

Suppose now that our cell $\Omega_{\delta}$ is embedded in an ambient medium $\mathcal{O}_{e}$, with conductivity equal to $\gamma_{e}$. We denote by

$$
\Omega=\Omega_{\delta} \cup \mathcal{O}_{e}
$$

and by $\gamma$ the function equal to $\gamma_{0}$ in $\mathcal{O}, \gamma_{1}$ in $\mathcal{O}_{\delta}$ and $\gamma_{e}$ in $\mathcal{O}_{e}$. Consider the steady-state voltage equations

$$
\left\{\begin{array}{lc}
\nabla \cdot(\gamma \nabla V)=0, & \text { in } \Omega \\
\frac{\partial V}{\partial n}=\phi, & \text { on } \partial \Omega
\end{array}\right.
$$

with $\phi$ as Neumann boundary condition on $\partial \Omega$.

We have to replace the membrane by approximate transmission conditions on the boundary of the cytoplasm. To obtain these conditions, we extend the exterior potential up to the boundary of the cytoplasm. Using the parametrization (3) of the membrane and Taylor expansions with respect to the normal variable, we then explicit the Dirichlet and Neumann values of the potential on the exterior boundary of the cell in terms of $\delta$ and of the Dirichlet and Neumann traces on the boundary of the cytoplasm. More precisely, if we denote by $V^{e}$ the potential in $\mathcal{O}_{e}$ formally extended to $\mathcal{O}_{\delta}$, and by $\Gamma_{\delta}$ the exterior boundary of the cell, we have

$$
\begin{aligned}
\left.V^{e}\right|_{\Gamma_{\delta}} & =\left.V^{e}\right|_{\partial \mathcal{O}}+\left.\left(x-\left.x\right|_{\partial \mathcal{O}}\right) \cdot n\left(\left.x\right|_{\partial \mathcal{O}}\right) \partial_{n} V^{e}\right|_{\partial \mathcal{O}}+\cdots, \\
\left.\partial_{n} V^{e}\right|_{\Gamma_{\delta}} & =\left.\partial_{n} V^{e}\right|_{\partial \mathcal{O}}+\left.\left(x-\left.x\right|_{\partial \mathcal{O}}\right) \cdot n\left(\left.x\right|_{\partial \mathcal{O}}\right) \partial_{n}^{2} V^{e}\right|_{\partial \mathcal{O}}+\cdots .
\end{aligned}
$$




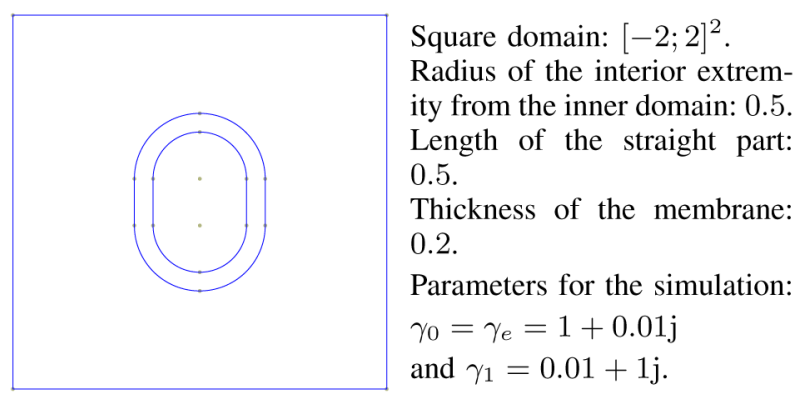

Fig. 4. The geometry considered: a cell embedded in a square domain. Representation with the thickest membrane.

We are now ready to apply the machinery of the asymptotic method presented in the previous section. We just give here the first two terms of the asymptotic expansion of $V$ solution to (14).

- Order 0. $V_{0}$ satisfies:

$$
\left\{\begin{array}{l}
\nabla \cdot\left(\gamma_{0} \nabla V_{0}\right)=0, \quad \text { in } \mathcal{O}, \\
\nabla \cdot\left(\gamma_{e} \nabla V_{0}\right)=0, \quad \text { in } \Omega \backslash \mathcal{O}, \\
\partial_{n} V_{0}=\phi \text { on } \partial \Omega \\
\text { with transmission conditions on } \partial \mathcal{O}: \\
\left.\gamma_{0} \partial_{n} V_{0}\right|_{\partial \mathcal{O}^{-}}=\left.\gamma_{e} \partial_{n} V_{0}\right|_{\partial \mathcal{O}^{+}}, \\
\left.V_{0}\right|_{\partial \mathcal{O}^{-}}=\left.V_{0}\right|_{\partial \mathcal{O}^{+}} .
\end{array}\right.
$$

- Order 1. $V_{1}$ satisfies

$$
\left\{\begin{array}{l}
\nabla \cdot\left(\gamma_{0} \nabla V_{1}\right)=0, \quad \text { in } \mathcal{O}, \\
\nabla \cdot\left(\gamma_{e} \nabla V_{1}\right)=0, \quad \text { in } \Omega \backslash \mathcal{O}, \\
\partial_{n} V_{e}=0 \text { on } \partial \Omega \\
\text { with transmission conditions on } \partial \mathcal{O}: \\
\left.\gamma_{0} \partial_{n} V_{1}\right|_{\partial \mathcal{O}^{-}}-\left.\gamma_{1} \partial_{n} V_{1}\right|_{\partial \mathcal{O}^{+}}=\left.\left(\gamma_{1}-\gamma_{e}\right) \partial_{t}^{2} V_{0}\right|_{\partial \mathcal{O}} \\
\left.V_{1}\right|_{\partial \mathcal{O}^{-}}-\left.V_{1}\right|_{\partial \mathcal{O}^{+}}=\left.\left(\frac{\gamma_{1}-\gamma_{e}}{\gamma_{1} \gamma_{e}}\right) \gamma_{0} \partial_{n} V_{0}\right|_{\partial \mathcal{O}^{-}}
\end{array}\right.
$$

The approximate potential $V_{\text {app }}$ is then the sum $V_{0}+\delta V_{1}$, and there exists a constant $C_{\gamma_{0}, \gamma_{1}, \gamma_{e}, \mathcal{O}} \delta$-independent such that

$$
\left\|V-V_{\text {app }}\right\|_{H^{1}(\mathcal{O})} \leq C_{\gamma_{0}, \gamma_{1}, \gamma_{e}, \mathcal{O}} \delta^{2}\|\phi\| .
$$

\section{B. Numerical Simulations}

An elongated cell embedded in a square domain is considered as shown in Fig. 4. The potential is enforced on the upper and lower boundaries; homogeneous Neumann conditions are considered for the lateral boundaries.

Fig. 5 shows a numerical validation of the approximate transmission conditions. Let us note that the calculated order is nonoptimal for the condition of order 1: an exponent of 1.7 is obtain instead of 2 as asserted in (17). As described before, it can be practically difficult to show numerically the estimate because the discretization error can be higher than what is asserted in (17).

\section{CONCLUSION}

We have presented how to build rigorously approximate conditions for the solution of the dielectric formulation in a domain with a thin membrane. With our method we do not have to mesh the membrane: the approximate field inside the thin layer is known explicitly, and the potential outside the membrane is

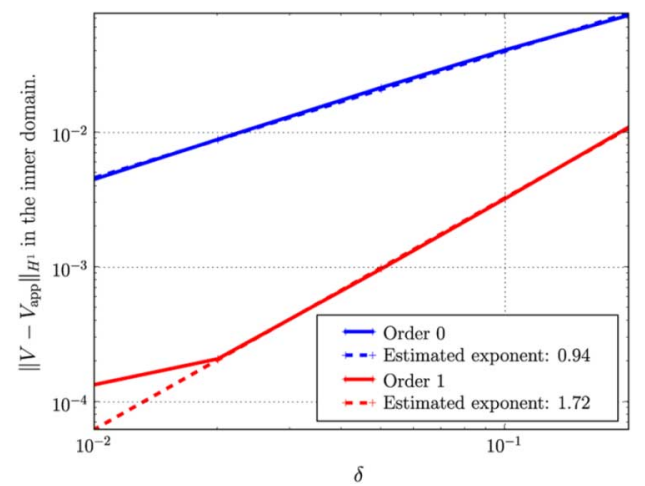

Fig. 5. Error versus the membrane thickness. $\gamma_{1}=0.01+1 \mathrm{j}, \gamma_{0}=\gamma_{e}=$ $1+0.01 \mathrm{j}$. The continuous lines are the results of the numerical computations and the dotted lines used an exponent estimated for (17) using the numerical results.

the solution to the dielectric formulation with the approximate condition. We provide rigorous a priori estimates of the error. Moreover, we may build approximate boundary condition at any order, if the domain and the boundary data are regular enough (see [6] and [7] for more details). This means that we may build solutions, which approach the total potential with an error in $\delta^{n}$, for $n$ as large as desired. The only drawback is that the weak formulation and the space of test functions for the problem with the approximate condition are less standard than in the original problem. This method has been generalized to the Helmholtz equation in [7] and it might be applied for the 3-D vector wave equation. It would also be useful to consider a domain with geometric singularities.

\section{REFERENCES}

[1] G. Pucihar, T. Kotnik, B. Valic, and D. Miklavcic, "Numerical determination of transmembrane voltage induced on irregularly shaped cells," Ann. Biomed. Eng., vol. 34, no. 4, pp. 642-652, 2006.

[2] J. Teissie, N. Eynard, B. Gabriel, and M. Rols, "Electropermeabilization of cell membranes," Adv. Drug Deliver Rev., vol. 35, pp. 3-19, 1999.

[3] M. Marty et al., "Electrochemotherapy-An easy, highly effective and safe treatment of cutaneous and subcutaneous metastases: Results of ESOPE (European Standard Operating Procedures of Electrochemotherapy) study," Eur. J. Cancer, no. 4, pp. 3-13, 2006.

[4] S. Munoz, J. Sebastian, M. Sancho, and J. Miranda, "Transmembrane voltage induced on altered erythrocyte shapes exposed to rf fields," Bioelectromagnetics, vol. 25, no. 1, pp. 631-633, 2004.

[5] E. Fear and M. Stuchly, "Modeling assemblies of biological cells exposed to electric fields," IEEE Trans. Biomed. Eng., vol. 45, no. 10, pp. 1259-1271, Oct. 1998, (electronic).

[6] C. Poignard, "Asymptotics for steady state voltage potentials in a bidimensionnal highly contrasted medium with thin layer," Math. Meth. Appl. Scl., Jul. 2007, doi: 10.1002/mma.923.

[7] C. Poignard, "Rigorous asymptotics for the elecric field in TM mode at mid-frequency in a bidimensional medium with thin layer," 2006, submitted [Online]. Available: http://www.cmap.polytechnique.fr/ poignard/helmholtz-27.html

[8] L. Krahenbiihl and D. Muller, "Thin layers in electrical engineering. Example of shell models in analysing eddy-currents by boundary and finite element methods," IEEE Trans. Magn., vol. 29, no. 2, pp. 1450-1455, Mar. 1993.

[9] P. Dular, C. Geuzaine, F. Henrotte, and W. Legros, "A general environment for the treatment of discrete problems and its application to the finite element method," IEEE Trans. Magn., vol. 34, no. 5, pp. 3395-3398, Sep. 1998.

Manuscript received June 24, 2007; revised November 1, 2007. Corresponding author: C. Poignard (e-mail: poignard@cmapx.polytechnique.fr). 